\title{
Evaluation of the Performance of Two Diagnostic Assays in Malaria Diagnosis in Mashonaland East Province, Zimbabwe, 2010
}

\author{
Regis C. Choto', Stanley M. Midzi², Joseph Mberikunashe², Mufuta Tshimanga1, \\ Notion T. Gombe1, Donewell Bangure ${ }^{{ }^{*}}$ \\ ${ }^{1}$ Department of Community Medicine, University of Zimbabwe, Harare, Zimbabwe \\ ${ }^{2}$ Ministry of Health and Child Care, Harare, Zimbabwe \\ Email: bangured@yahoo.com
}

Received 11 June 2015; accepted 31 July 2015; published 3 August 2015

Copyright $@ 2015$ by authors and Scientific Research Publishing Inc.

This work is licensed under the Creative Commons Attribution-NonCommercial International License (CC BY-NC).

http://creativecommons.org/licenses/by-nc/4.0/

(c) (i) (9) Open Access

\section{Abstract}

Introduction: Following the 2008 WHO in-vitro malaria RDT product testing study results, Paracheck RDT's sensitivity was revealed to significantly drop to $55 \%$ at low parasite densities. This raised concerns among health workers on its diagnostic capabilities and possible public health implications resulting from its continued use? We therefore evaluated the diagnostic performance of Paracheck and SD Bioline (a yet to be evaluated kit) RDTs under operational settings in areas of different endemicity. Methods: Using an analytic cross-sectional study design, finger prick blood samples from 422 clinically diagnosed patients selected from Mudzi (high malaria burden) and Murewa (low malaria burden) districts were each tested for malaria using Paracheck and SD Bioline RDTs and Giemsa stain microscopy as gold standard. Parasitemias were calculated using WHO standard protocols. Main outcomes were test efficiency; sensitivity; specificity; PVP and NPV. Results: Of eligible 390 blood slides prepared, microscopy detected malaria parasites in 125 $(32.1 \%)$ with $P$. falciparum being predominant species $(100 \%)$. Compared with microscopy, both Paracheck and SD Bioline RDTs performed fairly equally well and above WHO targets in Mudzi (high malaria burden), while in Murewa (low malaria burden) Paracheck and SD Bioline RDTs had sensitivities of $86.7 \%$ [69.5 - 100.0] and 86.7\% [86.7 - 100.0]; specificities of $97.2 \%$ [94.1 - 100.0] and 90.7\% [85.3 - 96.2]; test efficiencies of 95.9\% [92.4 - 99.4] and 90.2\% [85.0 - 95.5]; PVPs of $\mathbf{7 3 . 3 \%}$ and $\mathbf{4 5 . 2 \%}$; and NPVs of $\mathbf{9 8 . 8 \%}$ and $\mathbf{9 8 . 7 \%}$ respectively. Sensitivities for Paracheck and SD Bioline RDTs reduced from $99.2 \%$ [97.7 - 100.0] at parasitemias above $1000 / \mu \mathrm{l}$ each to $33.3 \%$ [0.0 - 71.1] and $50.0 \%$ [10.0 - 90.0] respectively at parasitemias below $1000 / \mu \mathrm{l}$ with variations not sta-

\footnotetext{
${ }^{*}$ Corresponding author.
}

How to cite this paper: Choto, R.C., Midzi, S.M., Mberikunashe, J., Tshimanga, M., Gombe, N.T. and Bangure, D. (2015) Evaluation of the Performance of Two Diagnostic Assays in Malaria Diagnosis in Mashonaland East Province, Zimbabwe, 2010. Open Journal of Epidemiology, 5, 187-196. http://dx.doi.org/10.4236/ojepi.2015.53023 
tistically significant. Conclusion: Paracheck RDT remains the MOHCC's preferred diagnostic alternative in areas where good quality microscopy is not available in Zimbabwe. SD Bioline RDT provides another diagnostic alternative especially in areas of high malaria burden. However performance of both kits at parasitemias below $1000 / \mu \mathrm{l}$ needs further investigation.

\title{
Keywords
}

\author{
Malaria Diagnosis, Mashonaland East, Zimbabwe
}

\section{Introduction}

Globally 250 - 300 million clinical malaria cases are recorded annually, and 90\% of these occur in developing countries, mainly in Sub-Saharan Africa. Annually 1.1 - 2.7 million people die from malaria, with more than a million being children under 5 years of age [1].

Beyond the human toll, malaria wreaks significant economic havoc. In high malaria burdened countries, the disease accounts for up to $40 \%$ of public health expenditures; $30 \%$ to $50 \%$ of inpatient hospital admissions and up to $60 \%$ of outpatient health clinic visits, resulting in reduction in economic growth rates by as much as $1.3 \%$ [2].

With the emergence of widespread chloroquine-resistant $P$. falciparum malaria in the early 1990s, the empirical use of chloroquine to treat suspected malaria became unsafe. The introduction of artemisinin combination therapies (ACTs) in Thailand in the mid-1990s and the adoption of ACTs as first-line treatment by most African countries by the early 2000s changed the cost-benefit ratio of empirical treatment of fever. The much higher cost of ACTs made specific diagnosis of malaria more cost-effective and demanded a more accurate diagnostic paradigm.

The World Health Organisation (WHO) recommended as a result, that malaria case management was based on parasite based diagnosis in all cases, with the exception of young children in areas of high transmission and where lack of resources or need for urgent response limits its application. While parasite based diagnosis of malaria has increased, most suspected cases are still not properly identified, with accurate diagnosis and disease monitoring consequently remains elusive.

Interest has thus spurred in developing diagnostic strategies that are effective not only in resource-limited areas, where malaria has a substantial burden on society, but also in developed countries where expertise in malaria diagnosis is often lacking [3] [4]. Though microscopic examination of stained blood smears remains the "gold standard" for detection of malaria parasitemia, its reliability is questionable, particularly at low levels of parasitemia and in the interpretation of mixed infection [5] [6]. In addition, it requires well-functioning equipment, highly trained staff and fresh reagents which in most situations are not available.

Recently, the development of antigen-detecting rapid diagnostic tests (RDTs) has offered the potential to provide accurate diagnosis to all at risk populations for the first time, reaching those unable to access good quality microscopy services. These tests are based on the detection of antigen(s) released from parasitized red blood cells [7]. The RDT signifies presence of these antigens by a colour change on an absorbing nitrocellulose strip. Some RDTs can detect only one species (Plasmodium falciparum), usually by detecting either histidine-rich protein-2(HRP-2) or parasite specific lactate dehydrogenase (pLDH). Some detect one or more of the other three species of malaria which infect humans, by detecting various other antigens.

RDTs commonly come in three different formats. The simplest format is a dipstick, which is placed in wells containing blood and/or buffer. The nitrocellulose strip may be placed in a plastic cassette or on a card. Cassettes and cards tend to be more expensive, but simpler to use. When in good condition, some products can achieve sensitivity similar to that commonly achieved by microscopy ( -100 parasites/ul).

To be widely useful, malaria RDTs must have adequate sensitivity (to ensure all clinically significant malaria infections are detected); specificity (to accurately discriminate non malarial febrile illness from malaria, to ensure appropriate management and accurate disease monitoring); stability (for accuracy to be maintained after transport and storage in ambient conditions) and ease of use and safety (to allow safe and correct preparation, and correct interpretation of results). Evaluations of malaria RDTs are done in terms of these 4 major requirements to assist national malaria control programs and other procurement agents to select products appropriate to 
their needs. Malaria RDTs' sensitivity varies between products. The recommended sensitivity is $\geq 95 \%$ at $\geq 100$ parasites/ul for $P$. falciparum [8].

In Zimbabwe, malaria remains one of the major public health challenges mainly as a result of weather patterns, which are conducive for mosquito breeding, and reduction in the flow of resources for the public health sector to implement basic public health interventions. It contributes to $20 \%-30 \%$ of the outpatient attendance and approximately 1.5 million clinical cases are reported annually [9].

The goal of the Zimbabwe National Malaria Control Program (NMCP) is to prevent mortality and reduce morbidity, social and economic losses due to malaria. To achieve this goal, the Zimbabwe NMCP committed itself to achieve set targets of the Abuja Declaration (reduce by 50\% of 2000 levels, malaria morbidity and mortality) and Millennium Development Goal (MDG) 6 (have halted, by 2015, and began to reverse the increasing incidence of malaria) by 2010 and 2015 respectively.

In 2006, Zimbabwe through Ministry of Health and Child Care, adopted a new malaria treatment policy, following sentinel drug efficacy monitoring studies which revealed an increased resistance by the malaria parasite to Chloroquine averaging 54\%, by early 2004. Under this new policy, laboratory confirmations of all suspected malaria cases by RDTs or microscopy at all levels become mandatory before dispensing treatment.

In an effort to make parasite based diagnosis of malaria accessible and affordable at all levels of the health delivery system, the MOHCC rolled out use of malaria RDTs in malaria diagnosis in 2006. Two malaria RDTs namely Paracheck RDT (an HRP-2 specific based test) and Optimal RDT (a Pan malaria antigen based test) were successfully evaluated in Zimbabwe and these were registered with the Laboratory Research Council of Zimbabwe. The Paracheck RDT kit was selected for national use over the Optimal RDT kit after consideration of its low cost and the fact that it detects Plasmodium falciparum malaria parasite-the predominant and severe form of malaria in Zimbabwe-accounting for $98 \%$ of malaria cases.

Ever since more new malaria RDTs have been developed with more interest being placed on the SD Bioline RDT-a relatively cheap Pan malarial antigen based test compared to Optimal RDTs, and other upcoming RDTs to diversify malaria diagnosis.

Since 2000, great strides have been made in Zimbabwe towards achieving 2010 Abuja targets through early diagnosis and prompt treatment of malaria; planning and implementing selective and sustainable preventive measures, including vector control; early detection, containment and prevention of epidemics; strengthening local capacities in basic and applied research and surveillance and IEC.

Mashonaland East province comprises of 9 administrative districts, with malaria transmission categorized into: sporadic or free malarious areas (Goromonzi, Wedza, Marondera, Seke and Chikomba districts); low endemic and seasonal (unstable) malarious areas (Murewa district); moderately endemic and seasonal (stable) malarious areas (Uzumba Maramba Pfungwe, Mtoko) and highly endemic and seasonal (stable) malarious areas (Mudzi).

Following publications by the World Health Organisation of results of an in-vitro product testing study of malaria RDTs in 2008, Paracheck RDT's sensitivity was noted to drop significantly at low parasite densities, with sensitivities reaching as low as 55\% at parasite densities of below 200 parasites/ul [10]. This has raised alarm, among health workers who are users of the kit and media circles, who are now questioning the diagnostic performance of the Paracheck P.f RDT kit in malaria detection and concerned about the possible public health implications resulting from its continued use?

If Paracheck RDT's sensitivity drops significantly at low parasite densities, its continued use is likely to have serious public health implications due to under diagnosis of a significant proportion of uncomplicated malaria cases, which may be missed, get complicated and result in more deaths. It will also not be consistent with WHO set validity prerequisites of at least $90 \%$ specificity and at least $95 \%$ sensitivity at parasite densities of at least $100 / \mathrm{ul}$, for any malaria RDTs to be used for the detection of malaria on a wide scale.

Considering also the fact that four years have lapsed since the last evaluation was done, Paracheck RDT kit and newly introduced rapid diagnostic tests need to be evaluated to diversify malaria diagnostic options. Results from such periodic evaluations will assist in guiding policy and future procurement decisions of RDTs by the MOHCC through the National Malaria Control Programme and ultimately improve malaria case management.

This study thus sought to assess the diagnostic performance of Paracheck P.f and SD Bioline malaria RDTs in two districts of Mashonaland East Province, specifically to determine and compare the malaria pick up rates and test efficiency of the kits.

\section{Methods}

An analytic cross-sectional study was conducted in Mudzi (high malaria burden) and Murewa (low malaria bur- 
den) districts of Mashonaland East province with five health institutions conveniently selected from the top 10 institutions in each district with highest incidences of malaria according to the 2008 and 2009 T5 surveillance statistics. These included Kotwa District Hospital; Nyamapanda; Kapotese; Dendera; Masarakufa clinics from Mudzi district and Murewa District Hospital; Nyamashato, Madamombe, Dandara and Goso clinics from Murewa district.

An uncomplicated malaria case was defined as any person living in malarious areas or who visited those areas within the last 6 weeks and presented with sudden onset of fever and any of the following: shivering and sweating, headache, nausea and vomiting. A severe malaria case was defined as any person living in malarious areas or who visited those areas within the last 6 weeks and presented with fever or a preceding history of fever and any signs of severe disease: impaired consciousness, fits/convulsions, jaundiced, coca cola urine, continuous vomiting, severe pallor and unexplained fever.

All patients with a clinical diagnosis of malaria who presented to the outpatients department of the participating health institutions during the period $9^{\text {th }}$ February 2010 to 5 March 2010 were eligible for enrolment into the study, if they were willing to participate and met the standard clinical malaria case definition and were resident in the respective district for at least 6 months

Assuming an error risk of 1.96, an expected Paracheck P.f RDT's sensitivity estimate of 50\%, (as reported by the WHO in an evaluation study on product testing of malaria RDTs in 2008) [10], an absolute precision of 5\%, plus an additional $10 \%$ of the sample to account for invalid and unclear results, a minimum sample size of 422 participants was recruited. Interviewer administered questionnaires were used to collect patient information on socio demographics, history of travel, prior drug history, symptoms and their duration. From each patient, a finger prick blood sample was collected for malaria testing with both Paracheck RDT and Giemsa stain microscopy.

Thick and thin films were prepared by a laboratory scientist, to ensure quality preparation of slides. Standard microscopy was performed on thick blood films of all samples to diagnose malaria and to assess parasite density, and on thin blood films of positive samples to define the Plasmodium species. Parasitemia was calculated according to World Health Organisation standard protocols. Slide interpretations were done by 2 microscopists who had no knowledge of each other's findings and the patient's immunochromatographic test result (to avoid bias). Discordant results were checked by a third microscopist who was blinded from microscopy and immunochromatographic test result findings by the other microscopists.

Immunochromatographic testing, with Paracheck P.f and SD Bioline RDT kits, was performed according to the manufacturer's instructions. Same batch numbers for RDTs were used for each district. RDT strip line intensities were graded based on visual assessment by 2 independent readers as "strong" or "faint positive" for reactive tests and "negative" for non-reactive ones. A test result was graded "strong" if the test line was as intense as the control line and "faint" if test line could only be seen in good light.

Epi info software package (Version 3.5.1-August 2008) was used to conduct statistical analyses of quantitative data to ascertain the average parasitemias and to determine malaria pick up rates of the Paracheck P.f RDT, SD Bioline RDT and microscopy for each district. Sensitivities, specificities, test efficiencies and their 95\% CIs, positive predictive values and negative predictive values were calculated for each district.

Permission to carry out the study was sought from the Provincial Medical Directorate (PMD) Mashonaland East Province, District Medical Office (DMO) for Mudzi and Murewa Districts, and the Health Studies Office. Ethical clearance was sought from the Medical Research Institute of Zimbabwe, MOHCC. Confidentiality was assured and maintained throughout the study and the aim of the study was explained to all potential participants and written consent was sought. Blood was collected after informed consent was obtained from all adult participants at the field site, and from guardians of all minors. The participants were informed that they were free to withdraw at any time without giving reasons. Decision not to participate was respected and the participants were assured that non participation was not going to affect their health care in any way.

Physical examinations were performed on confirmed malaria negative cases (based on Paracheck P.f RDT and/or SD Bioline RDT and/or microscopy results) for differential diagnosis and these were managed appropriately. Confirmed malaria cases per microscopy or Paracheck P.f RDT or SD Bioline RDT kit result were managed based on the new national malaria treatment guidelines.

\section{Results}

Out of the total 422 suspected cases enrolled in the study, thirty-two cases were excluded due to invalid results 
or breakages of prepared blood slide smears during analysis. The median age of the remaining 390 suspected malaria cases was 17 years $\left(\mathrm{Q}_{1}=8\right.$ years; $\left.\mathrm{Q}_{3}=32\right)$ with a distribution of 157 males and 233 females in the ratio of $1: 1.5$. Age groups above 15 years of age were the most affected accounting for $60.8 \%$ of the total confirmed cases.

The majority of confirmed cases (56.8\%) presented at the health centre 1 - 3 days after onset of illness. Fever with associated headache and/or chills and rigors and/or nausea and vomiting were the most common presenting symptoms accounting for at least $30 \%$ among confirmed cases.

Of the resulting 390 blood smear (thick/thin) slides eligible for analysis, microscopy detected malaria parasites overall in 125 (32.1\%) blood smear samples: 110/267 (41.2\%) blood smear samples in Mudzi-a high burdened malaria district and 15/123 (12.2\%) in Murewa-a low burdened malaria district. Compared to microscopy, SD Bioline RDT detected malaria antigens in 139 (35.6\%) blood smear samples: 116 (43.4\%) blood smear samples in Mudzi district and 23 (18.7\%) blood smear samples in Murewa district. Paracheck RDT on the other hand, detected malaria antigens overall in 128 (32.8\%) blood smear samples: 112 (41.9\%) blood smear samples in Mudzi district and 16 (13.0\%) blood smear samples in Murewa district. Table 1 illustrates results of the detection of malaria by the two rapid antigen detection tests in comparison with microscopy.

Table 1. Malaria detection rates of microscopy, Paracheck P.f and SD Bioline rapid diagnostic approaches in Mashonaland east, Zimbabwe.

\begin{tabular}{cccc}
\hline & \multicolumn{3}{c}{ Malaria Detection Rates } \\
\hline Method & Combined Districts $\mathrm{n}=390(\%)$ & Mudzi District $\mathrm{n}=267(\%)$ & Murewa District $\mathrm{n}=123(\%)$ \\
\hline Microscopy & $125(32.1)$ & $110(41.2)$ & $15(12.2)$ \\
Paracheck RDT & $128(32.8)$ & $112(41.9)$ & $16(13.0)$ \\
SD Bioline RDT & $139(35.6)$ & $116(43.4)$ & $23(18.7)$ \\
\hline
\end{tabular}

Overall the median parasite density for confirmed cases in the 2 districts was 8520 parasites/ul $(4000,15,440)$ with the median parasite density for confirmed cases in Mudzi district (high malaria burden) being 8600 parasites/ul (4000; 16,000) while that for Murewa district (low malaria burden) was 8400 parasites/ul (3400; 9500) illustrating no significant difference in parasitaemia between the 2 districts.

Compared with microscopy, both tests overall performed fairly well in malaria diagnosis with Paracheck RDT attaining a sensitivity of 96.0\% [92.6 - 99.4]; specificity of 97.0\% [94.9 - 99.0]; test efficiency of 96.7\% [94.9 98.4]; predictive value positive (PVP) of $83.7 \%$ and negative predictive value (NPV) of 99.3\%, while SD Bioline RDT had a sensitivity of $96.8 \%$ [93.7 - 99.9]; specificity of $93.2 \%$ [90.2 - 96.2]; test efficiency of $94.4 \%$ [92.1 - 96.6]; predictive value positive (PVP) of 69.7\% and negative predictive value (NPV) of 99.4\%.

On stratifying diagnostic performance of the 2 tests by area of residence, both tests performed almost equally well in Mudzi-a high malaria burdened district, with performance indicators well above the WHO recommended targets of at least a sensitivity of $95 \%$ and specificity of at least $90 \%$. In Murewa-a low burdened malaria district, Paracheck RDT had higher specificity; test efficiency; PVP and NVP compared to the SD Bioline RDT. Notably different for both tests was the predictive value positive (PVP) with Paracheck RDT attaining a PVP of 73.3\% compared to the SD Bioline RDT which had a PVP of $45.2 \%$. Table 2 below illustrates the diagnostic performance of the 2 tests by area of residence.

Table 2. Diagnostic performance of paracheck and SD bioline RDTs by area of residence.

\begin{tabular}{|c|c|c|c|c|c|c|}
\hline \multirow{2}{*}{ Area } & \multirow[b]{2}{*}{ Method } & \multicolumn{5}{|c|}{ Diagnostic Performance } \\
\hline & & Sensitivity \% CI & Specificity \% CI & Test Efficiency \% CI & PPV \% & NPV \% \\
\hline \multirow[t]{2}{*}{ Mudzi } & Paracheck & $97.3[94.2-100.0]^{*}$ & 96.8 [94.1 - 99.6] & $97.0[95.0-99.0]$ & 88.2 & 99.3 \\
\hline & SD Bioline & $98.2[95.7-100.0]^{*}$ & 94.9 [91.5 - 98.3] & $96.3[94.0-98.5]$ & 82.5 & 99.5 \\
\hline \multirow[t]{2}{*}{ Murewa } & Paracheck & $86.7[69.5-100.0]^{*}$ & $97.2[94.1-100.0]^{*}$ & 95.9 [92.4 - 99.4] & 73.3 & 98.8 \\
\hline & SD Bioline & $86.7[69.5-100.0]^{*}$ & $90.7[85.3-96.2]$ & $90.2[85.0-95.5]$ & 45.2 & 98.7 \\
\hline \multirow[t]{2}{*}{ Combined } & Paracheck & $96.0[92.6-99.4]$ & $97.0[94.9-99.0]$ & 96.7 [94.9 - 98.4] & 83.7 & 99.3 \\
\hline & SD Bioline & 96.8 [93.7 - 99.9] & $93.2[90.2-96.2]$ & $94.4[92.1$ - 96.6] & 69.7 & 99.4 \\
\hline
\end{tabular}

${ }^{*}$ Confidence intervals rounded off to fall between 0 and 100. 
On analysis of the diagnostic performance of both tests by parasite density, sensitivities of both Paracheck and SD Bioline RDTs were noted to be almost equally affected by variations in parasite densities with sensitivity directly related to the degree of parasitemia. Assessment of sensitivities of both tests at parasitemic thresholds below 1000 were however not possible due to the very small sample sizes involved in our study. Table 3 highlights the association between sensitivity of both kits and parasite density.

Table 3. Diagnostic performance of Paracheck and SD Bioline RDTs Matched by parasite density, Mashanaland east, Zimbabwe.

\begin{tabular}{|c|c|c|c|c|}
\hline $\begin{array}{l}\text { Microscopy Confirmed } \\
\text { Samples Tested }\end{array}$ & $\begin{array}{l}\text { Parasite Density } \\
\text { (parasites/ul) }\end{array}$ & Diagnostic Method Used & $\begin{array}{l}\text { Number of Samples that } \\
\text { Tested Positive }\end{array}$ & $\begin{array}{l}\text { Sensitivity } \\
\text { \% CI }\end{array}$ \\
\hline \multirow{2}{*}{6} & \multirow{2}{*}{$<1000$} & Paracheck RDT & 2 & $33.3[0.0-71.1]^{*}$ \\
\hline & & SD Bioline RDT & 3 & $50.0[10.0-90.0]$ \\
\hline \multirow{2}{*}{119} & \multirow{2}{*}{$>1000$} & Paracheck RDT & 118 & $92.2[97.5-100.0]^{*}$ \\
\hline & & SD Bioline RDT & 118 & $99.2\left[[97.5-100.0]^{*}\right.$ \\
\hline \multirow{2}{*}{20} & \multirow{2}{*}{$<2500$} & Paracheck RDT & 16 & $80.0[62.5-97.5]$ \\
\hline & & SD Bioline RDT & 17 & $85.0[69.4-100.0]^{*}$ \\
\hline \multirow{2}{*}{105} & \multirow{2}{*}{$>2500$} & Paracheck RDT & 104 & $99.1[97.2-100.0]^{*}$ \\
\hline & & SD Bioline RDT & 104 & $99.1[97.2-100.0]^{*}$ \\
\hline \multirow{2}{*}{37} & \multirow{2}{*}{$<5000$} & Paracheck RDT & 33 & $89.2[79.2-99.2]$ \\
\hline & & SD Bioline RDT & 34 & $91.9[83.1-100.0]^{*}$ \\
\hline \multirow{2}{*}{88} & \multirow{2}{*}{$>5000$} & Paracheck RDT & 87 & $98.9[96.6-100.0]^{*}$ \\
\hline & & SD Bioline RDT & 87 & $98.9[96.6-100.0]^{*}$ \\
\hline
\end{tabular}

${ }^{*}$ Confidence intervals rounded off to fall between 0 and 100.

Out of the total 125 blood smear samples detected as positive by microscopy, Paracheck and SD Bioline RDTs generated strong line intensities in 102 (81.6\%) and 113 (90.4\%) samples respectively; faint line intensities in $18(14.4 \%)$ and 8 (6.4\%) samples respectively and negative test lines in $5(4.0 \%)$ and 4 (3.2\%) samplesrespectively. Strong line intensities occurred only at high parasite densities of above 1000 parasites/ul. In contrast, faint line intensities for both Paracheck and SD Bioline tests occurred not only at relatively low parasite densities of below 1000/ $\mu$ l, but also at relatively high parasite densities above 1000/ul. Both Paracheck and SD Bioline tests failed to detect malaria infections in 5/125 (4.0\%) and 4/125 (3.2\%) microscopically confirmed blood smear samples respectively. Notably for both tests these "false negative" $P$. falciparum blood smear samples they failed to detect, showed no particular geographic distribution and most of them (4/5 for Paracheck test and 3/4 for SD Bioline test) had relatively low parasite densities of below 1 000/ul. Table 4 shows variations of the intensity of the positivity bands matched with parasite density for the two RDT devices

Table 4. Intensity of the Paracheck/SD Bioline RDT’s Positivity Bands Matched with Parasite Density, Mashonaland East, Zimbabwe.

\begin{tabular}{|c|c|c|c|c|}
\hline \multirow{2}{*}{ Parasite Density } & \multirow{2}{*}{$\begin{array}{l}\text { Specimens with Indicated Density } \\
\text { as Determined by }\end{array}$} & \multicolumn{3}{|c|}{ Consensus Reading of Paracheck/SD Positivity Bands } \\
\hline & & Negative $\left(\mathbf{n}_{1}\right)$ & Faint $\left(\mathbf{n}_{2}\right)$ & Strong $\left(\mathbf{n}_{3}\right)$ \\
\hline \multirow[t]{2}{*}{$0-500 /$ ul (+) } & Paracheck RDT & $2(1.6)$ & $0(0.0)$ & $0(0.0)$ \\
\hline & SD Bioline RDT & $2(1.6)$ & $0(0.0)$ & $0(0.0)$ \\
\hline \multirow[t]{2}{*}{501 - 1000/ul (++) } & Paracheck RDT & $2(1.6)$ & $2(1.6)$ & $0(0.0)$ \\
\hline & SD Bioline RDT & $1(0.8)$ & $2(1.6)$ & $0(0.0)$ \\
\hline \multirow[t]{2}{*}{$1001-5000 / \mathrm{ul}(+++)$} & Paracheck RDT & $0(0.0)$ & $5(4.0)$ & $26(20.8)$ \\
\hline & SD Bioline RDT & $0(0.0)$ & $3(2.4)$ & $28(22.4)$ \\
\hline \multirow[t]{2}{*}{$>5000 /$ ul $(++++)$} & Paracheck RDT & $1(0.8)$ & $11(8.8)$ & $76(60.8)$ \\
\hline & SD Bioline RDT & $1(0.8)$ & $3(2.4)$ & $85(68.0)$ \\
\hline
\end{tabular}

N.B: Faint positivity band on the SD Bioline RDT cassette referred to faint P.f and/or Pan lines. 
Of the total 390 blood smears examined, 11 were picked as "false positives" and 5 as "false negatives" by the Paracheck RDT kit. The "false-negative" $P$. falciparum samples showed no particular geographic distribution and most of them (3/5) had relatively low parasite densities (more than 5000/ $\mu$ l). The 11 "false positive" P. falciparum samples generated both weak and strong line intensities on the Paracheck RDT cassette; with the majority (8/11) coming from Mudzi district.

\section{Discussion}

Against a background of limited evaluations done in Zimbabwe on performance of malaria RDTs in low malaria burdened areas, this study provided an opportunity to evaluate two malaria RDTs (one currently in use nationally-Paracheck RDT and the other a new assay yet to be evaluated-SD Bioline RDT) in an operational setting in areas of different malaria endemicity.

Key findings from the evaluation supported the possible use of both Paracheck P.f and SD Bioline RDTs as good alternatives to malaria diagnosis where good quality microscopy is not available as shown by comparable malaria parasitological confirmations by the two diagnostic assays (32.8\% \& $35.6 \%$ ) to that of microscopy (32.1\%) from the same study population. This avails more options to the national programme in malaria diagnosis and ultimately mitigates extra costs incurred through unnecessary treatment of non-malarial fevers by Artemisinin-based Combination Therapies (ACTs) due to use of clinical based diagnosis in areas where microscopy is not available. In addition, this reduces unnecessary exposure to potential adverse effects of ACTs and prevents the spread of parasite resistance to these drugs, which has already been reported in South East Asia [11]-[13].

In our study findings, more infections (inclusive of "false positives") were detected by both the Paracheck and the SD Bioline RDT kits than by blood slide microscopy in both regions. This is in contrast to findings by Endeshaw $\mathrm{T}$ et al. in 2008, who observed that microscopy tends to pick more malaria infections in low parasite density areas compared to malaria RDTs while in high parasite density areas, malaria RDTs tend to pick up more infections than microscopy. ${ }^{31}$ The noted discrepancies with our study findings may be explained by the fact that median parasite densities between the 2 districts were relatively comparable. In addition, the last stratification exercise to map the malaria burden in Zimbabwe was done in 2002 which may be illustrating the changes that may have occurred since then due to global warming and highlights the need to re-stratify malaria burden in Zimbabwe.

The very high overall sensitivities of both malaria RDTs (Paracheck and SD Bioline) of at least 96.0\% implies that the majority of malaria cases among this population were accurately diagnosed by both tests, giving the clinician the confidence that true cases of malaria were rarely missed. Related to the high sensitivities, Paracheck and SD Bioline RDTs also demonstrated overall, very high Negative Predictive Values in this study population which allowed clinicians to confidently diagnose negative test patients by either test, as non-malaria patients and look for other causes of fever. The averted non malaria treatment leads to cost saving and improves quality of care.

Even though performance of both malaria RDT kits was noted to be significantly influenced by level of parasitemia (with sensitivities of Paracheck and SD Bioline RDTs dropping from 99.2\% [97.7 - 100.0] ${ }^{*}$ at parasitemias of above $1000 / \mu l$ to $33.3 \%$ [0.0 - 71.1] $]^{*}$ and 50.0\% [10.0 - 90.0] respectively at parasitemias of below

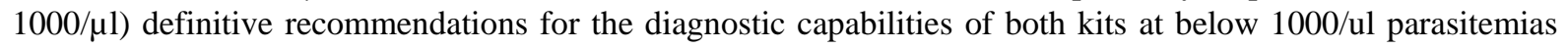
cannot be made due to a small sample size of specimens in this category seen. Under diagnosis of such cases can potentially be dangerous, as in most cases these patients will be relatively well except for the fever resulting in them complicating because appropriate treatment may not have been instituted in time. The assessment of a negative result in this situation will be clearly influenced by the clinical features.

Overall, the SD Bioline RDT detected slightly more "infections" than Paracheck RDT explaining the differences in the positive predictive values between the 2 diagnostic assays of $69.7 \%$ and $83.7 \%$ respectively. This would mean overall, out of a population of patients with true malarial disease, the Paracheck RDT performs better in detecting malarial antigens by $14.0 \%$ compared to the SD Bioline RDT kit. On stratification by area of residence, both tests kits interestingly were noted to perform almost the same in detecting true cases of malaria in the high burdened malaria district (Mudzi). Notably however in the low burdened malaria district (Murewa) Paracheck RDT was noted to perform better by $28.1 \%$ compared to the SD Bioline RDT kit. As a result it can be concluded that the both kits perform almost the same in detecting true malaria cases in areas of high malaria burden but in areas of low malaria burden the Paracheck RDT provides a better test compared to the SD Bioline 
RDT kit in detecting true cases.

However "false-positive" results appear to play a minor role in evaluating the usefulness of malaria tests for clinical settings. False-positive reactions may occur in individuals who have been recently treated for malaria or if patients have circulating rheumatoid factors [14]-[16]. Preliminary data from studies done elsewhere show that the PfHRP-2 antigen (detected in this situation by both the Paracheck P.f and SD Bioline RDTs) may persist for up to 7 to 10 days after asexual parasite clearance [17] [18] whereas, circulating pLDH activity (detected by the SD Bioline P.f/Pan test only) drops profoundly immediately after the parasites are cleared from the peripheral blood [19].

This has led to the recommendation of pLDH based RDTs as suitable tests for treatment monitoring [19]-[21]. However, pLDH is also produced by gametocytes [20], which are not affected by many antimalarial drugs and may thus be present long after asexual parasitemia has been cleared and the patient has recovered. The presence of gametocytes could therefore greatly reduce the utility of pLDH-based tests [22].

In our study, more false positives were picked by the SD Bioline RDT-both an HRP-2-based and pLDH based-test, than by the Paracheck RDT-a HRP-2 based antigen based test. This may be explained by the probable presence of gametocytemia in addition to the persistence of HRP-2 antigen. On the other hand, it may also be explained by the fact that most of the false-positive cases may have been "true positives" which were not detected by microscopy, due to sequestration limiting the number of circulating parasites at the time of blood collection or due to the parasitemia being below the detection limit of approximately 50/ $\mu$ by microscopy.

Paracheck and SD Bioline RDTs picked 5 and 4 "false negative" results respectively with the majority of these (4/5 for Paracheck RDT and 3/4 for SD Bioline RDT) occurring at parasite densities of below 1000/ul and the remainder occurring at parasite densities of more than 5000/ul. A false negative result where parasite density is high has been described by other investigators as a very rare event which may be caused by a prozone effect or by the presence of a mutation or deletion within the HRP-2 gene [23]. This also implies the need for a continuous quality assurance system to be instituted at every step before disseminating the RDT products to end users.

The majority of samples which generated "false negative" results or faint lines on Paracheck and/or SD Bioline RDT cassettes occurred at relatively low parasite densities i.e. less than 5000/ul. Faint test lines are a frequently described problem, which may be caused by the fact that the test line is much narrower than the control line [24] [25].

This phenomenon is important especially in field conditions where interpretation of faint positive lines as negative test results is found as the most common mistake made by community health care workers even when provided with adapted job aids and problems can be expected when reading RDTs during evening and night shifts, particularly for readers with poor visual capacities [26]-[28]. Most "false negative" results in malaria RDTs are linked to low parasitemias which in most situations are unlikely to be the primary cause of illness.

\section{Conclusions}

Both Paracheck and SD Bioline RDTs provide accurate and reliable diagnostic alternatives where good quality microscopy may not be available in both Mudzi (high malaria burden) and Murewa (low malaria burden) districts of Mashonaland East province. Both Paracheck and SD Bioline RDT kits perform almost similarly well in detecting true malaria cases in areas of high malaria endemicity while in low malaria burdened areas the Paracheck RDT kit provides a better test than the SD Bioline RDT. However their diagnostic performances are affected by variations in parasite densities with their sensitivities at parasite densities below $1000 / \mu 1$ still needing further investigation.

Thick blood film examination however remains the standard method for diagnosing malaria because it detects all Plasmodium species and offers the clear distinctions between parasite growth stages, which are essential for therapeutic decisions.

\section{Competing Interests}

The authors declare no competing interest.

\section{Authors' Contributions}

Regis C. Choto was responsible for the conception of the problem, design, collection, analysis and interpretation 
and drafting of the final article. Notion T. Gombe was responsible for conception of the problem, design, analysis and interpretation of data and drafting the final article. Donewell Bangure was responsible for conception of the problem, design, analysis and interpretation of data and drafting the final article. Joseph Mberikunashe was responsible for conception of the problem, design, analysis and interpretation of data and drafting the final article. Stanley M. Midzi was responsible for conception of the problem, design, analysis and interpretation of data and drafting the final article. Mufuta Tshimanga had oversight of all stages of the research and critically reviewed the final draft for academic content. The manuscript was read and approved by all authors.

\section{Acknowledgements}

I wish to acknowledge the support I received from my academic and field supervisors, the National Microbiology Reference Laboratory (Dr. S. Zinyowera and her staff) whose contribution to the successful development and actual carrying out of this project was invaluable. Special thank you also goes to the PMD, Mashonaland East province (Dr Zizhou), DMOs for Mudzi and Murewa districts and their health staff, my wife and family and all participants who partook in the study. To the MOHCC through the NMCP, the Health Studies Office (HSO), the Department of Community Medicine, University of Zimbabwe and the Centre for Disease Control and Prevention (CDC-Atlanta) your support in making this project a reality is greatly appreciated.

\section{References}

[1] WHO (2008) World Malaria Report. Geneva.

[2] WHO (2015) Malaria Factsheet No. 95. http://www.who.int/mediacentre/factsheets/fs094/en/index.html

[3] Bell, D., Wongsrichanalai, C. and Barnwell, J.W. (2006) Ensuring Quality and Access for Malaria Diagnosis: How Can It Be Achieved? Nature Reviews Microbiology, 4, S7-S20. http://dx.doi.org/10.1038/nrmicro1525

[4] Reyburn, H., Mbakilwa, H., Mwangi, R., Mwerinde, O., Olomi, R., et al. (2007) Rapid Diagnostic Tests Compared with Malaria Microscopy for Guiding Outpatient Treatment of Febrile Illness in Tanzania: Randomised Trial. BMJ, 334, 403. http://dx.doi.org/10.1136/bmj.39073.496829.AE

[5] Molyneux, M. and Fox, R. (1993) Diagnosis and Treatment of Malaria in Britain. BMJ, 306, 1175-1180. http://dx.doi.org/10.1136/bmj.306.6886.1175

[6] World Health Organization (1996) Management of Uncomplicated Malaria and the Use of Antimalarial Drugs for the Protection of Travellers. Report of an Informal Consultation. W.H.O./MAL/96. World Health Organization, Geneva, 98.

[7] Moody, A.H. and Chiodini, P.L. (2002) Non-Microscopic Method for Malaria Diagnosis Using OptiMAL IT, a Second-Generation Dipstick for Malaria pLDH Antigen Detection. British Journal of Biomedical Science, 59, $228-231$.

[8] WHO (2006) The Use of Malaria Rapid Diagnostic Tests. 2nd Edition.

[9] (2006) Zimbabwe Demographic Health Survey.

[10] WHO (2008) Malaria Rapid Diagnostic Test Performance. Results of WHO Product Testing of Malaria RDTs: Round 1.

[11] Dondorp, A.M., Nosten, F., Yi, P., Das, D., Phyo, A.P., et al. (2009) Artemisinin Resistance in Plasmodium falciparum Malaria. The New England Journal of Medicine, 361, 455-467. http://dx.doi.org/10.1056/NEJMoa0808859

[12] Carrara, V.I., Zwang, J., Ashley, E.A., Price, R.N., Stepniewska, K., et al. (2009) Changes in the Treatment Responses to Artesunate-Mefloquine on the Northwestern Border of Thailand during 13 Years of Continuous Deployment. PLoS ONE, 4, e4551. http://dx.doi.org/10.1371/journal.pone.0004551

[13] Noedl, H., Socheat, D. and Satimai, W. (2009) Artemisinin-Resistant Malaria in Asia. The New England Journal of Medicine, 361, 540-541. http://dx.doi.org/10.1056/NEJMc0900231

[14] Beadle, C., Long, G.W., Weiss, W.R., McElroy, P.D., Maret, S.M., et al. (1994) Diagnosis of Malaria by Detection of Plasmodium falciparum HRP-2 Antigen with a Rapid Dipstick Antigen-Capture Assay. Lancet, 343, 564-568. http://dx.doi.org/10.1016/S0140-6736(94)91520-2

[15] Singh, N., Valecha, N. and Sharma, V.P. (1997) Malaria Diagnosis by Field Workers Using an Immunochromatographic Test. Transactions of the Royal Society of Tropical Medicine and Hygiene, 91, 396-397. http://dx.doi.org/10.1016/S0035-9203(97)90254-6

[16] Jelinek, T., Grobusch, M.P. and Harms, G. (2001) Evaluation of a Dipstick Test for the Rapid Diagnosis of Imported Malaria among Patients Presenting within the Network. Scandinavian Journal of Infectious Diseases, 33, 752-754. 
http://dx.doi.org/10.1080/003655401317074563

[17] Eisen, D.P. and Saul, A. (2000) Disappearance of Pan-Malarial Antigen Reactivity Using the ICT Malaria Pf/Pv Kit Parallels Decline of Patent Parasitemia as Shown by Microscopy. Transactions of the Royal Society of Tropical Medicine and Hygiene, 94, 169-170. http://dx.doi.org/10.1016/S0035-9203(00)90262-1

[18] Shiff, C.J., Premji, Z. and Minjas, J.N. (1993) The Rapid Manual ParaSight ${ }^{\circledR}$-F Test: A New Diagnostic Tool for Plasmodium falciparum Infection. Transactions of the Royal Society of Tropical Medicine and Hygiene, 87, 646-648. http://dx.doi.org/10.1016/0035-9203(93)90273-S

[19] Piper, R., Lebras, J., Wentworth, L., Hunt-Cooke, A., Houze, S., et al. (1999) Immunocapture Diagnostic Assays for Malaria Using Plasmodium Lactate Dehydrogenase (pLDH). The American Journal of Tropical Medicine and Hygiene, 60, 109-118.

[20] Oduola, A.M., Omitowoju, G.O., Sowunmi, A., Makler, M.T., Falade, C.O., et al. (1997) Plasmodium falciparum: Evaluation of Lactate Dehydrogenase in Monitoring Therapeutic Responses to Standard Antimalarial Drugs in Nigeria. Experimental Parasitology, 87, 283-289. [PubMed]. http://dx.doi.org/10.1006/expr.1997.4251

[21] Singh, N., Valecha, N., Nagpal, N.A.C., Mishra, S.S., Varma, H.S. and Subbarao, S.K. (2003) The Hospital- and Field-Based Performances of the OptiMAL Test, for Malaria Diagnosis and Treatment Monitoring in Central India. Annals of Tropical Medicine and Parasitology, 97, 5-13. http://dx.doi.org/10.1179/000349803125002544

[22] Tjitra, E. and Anstey, N.M. (2001) Will the High Rates of Post-Treatment Sexual Stage Parasitaemia Seen in Malaria-Endemic Areas Make the OptiMAL Antigen Test Unreliable in Predicting Malaria Treatment Outcome? British Journal of Haematology, 113, 255-257. http://dx.doi.org/10.1046/j.1365-2141.2001.02673-2.x

[23] Pieroni, P., Mills, C.D., Ohrt, C., Harrington, M.A. and Kain, K.C. (1998) Comparison of the ParaSight-F Test and the ICT Malaria Pf Test with the Polymerase Chain Reaction for the Diagnosis of Plasmodium falciparum Malaria in Travellers. Transactions of the Royal Society of Tropical Medicine and Hygiene, 92, 166-169. http://dx.doi.org/10.1016/S0035-9203(98)90730-1

[24] www.wpro.who.int/sites/rdt/documents

[25] Craig, M.H., Bredenkamp, B.L., Williams, C.H., Rossouw, E.J., Kelly, V.J., et al. (2002) Field and Laboratory Comparative Evaluation of ten Rapid Malaria Diagnostic Tests. Transactions of the Royal Society of Tropical Medicine and Hygiene, 96, 258-265. http://dx.doi.org/10.1016/S0035-9203(02)90092-1

[26] McMorrow, M.L., Masanja, M.I., Abdulla, S.M., Kahigwa, E. and Kachur, S.P. (2008) Challenges in Routine Implementation and Quality Control of Rapid Diagnostic Tests for Malaria—Rufiji District, Tanzania. The American Journal of Tropical Medicine and Hygiene, 79, 385-390.

[27] Mayxay, M., Pukrittayakamee, S., Newton, P.N. and White, N.J. (2004) Mixed-Species Malaria Infections in Humans. Trends in Parasitology, 20, 233-240. http://dx.doi.org/10.1016/j.pt.2004.03.006

[28] Harvey, S.A., Jennings, L., Chinyama, M., Masaninga, F., Mulholland, K., et al. (2008) Improving Community Health Worker Use of Malaria Rapid Diagnostic Tests in Zambia: Package Instructions, Job Aid and Job Aid-Plus-Training. Malaria Journal, 7, 160. http://dx.doi.org/10.1186/1475-2875-7-160 